\title{
MoKi: The Enterprise Modelling Wiki
}

\author{
Chiara Ghidini ${ }^{1}$, Barbara Kump ${ }^{2}$, Stefanie Lindstaedt ${ }^{2,3}$, Nahid Mahbub ${ }^{1}$, \\ Viktoria Pammer ${ }^{2,3}$, Marco Rospocher ${ }^{1}$, and Luciano Serafini ${ }^{1}$ \\ 1 FBK-irst. Via Sommarive 18 Povo, 38050, Trento, Italy \\ 2 Knowledge Management Institute, TU Graz. Inffeldgasse 21a, 8010 Graz, Austria \\ 3 Know-Center. Inffeldgasse 21a, 8010 Graz, Austria
}

\begin{abstract}
Enterprise modelling focuses on the construction of a structured description, the so-called enterprise model, which represents aspects relevant to the activity of an enterprise. Although it has become clearer recently that enterprise modelling is a collaborative activity, involving a large number of people, most of the enterprise modelling tools still only support very limited degrees of collaboration. Within this contribution we describe a tool for enterprise modelling, called MoKi (MOdelling wiKI), which supports agile collaboration between all different actors involved in the enterprise modelling activities. MoKi is based on a Semantic Wiki and enables actors with different expertise to develop an enterprise model not only using structural (formal) descriptions but also adopting more informal and semi-formal descriptions of knowledge.
\end{abstract}

\section{Research Background: Enterprise Modelling}

According to 1], "an enterprise model is a computational representation of the structure, activities, processes, information, resources, people, behavior, goals, and constraints of a business, government, or other enterprise". We refer to enterprise modelling as the process of creating an enterprise model. Often, an enterprise model focuses on the description of two specific aspects of an enterprise: (i) its processes and activities, and / or (ii) the business domain within which the enterprise operates. Additionally, other aspects of an enterprise, like goals, human resources, organisational structure and roles etc. may also be important assets to be described in an enterprise model. This is due to the central role that enterprise models are playing in the development of a large number of applications, including Internet and (Semantic) Web based applications.

Building an enterprise model requires a number of skills. These skills span from knowing the different aspects that have to be described in the models, to having the ability of encoding such knowledge into formal statements, to having the ability of integrating different aspects, such as structure, activities, processes, information, resources, people, behavior, goals, and constraints into a uniform and coherent vision. Given the complexity of enterprise modelling, it is unrealistic to assume that all the actors possess all the skills above. For instance different actors may have very different expertise in encoding some content into formal languages, or may know only of specific aspects of an enterprise. For this 
reason, enterprise modelling is inherently a collaborative activity, performed by different actors with different know-how and technical skills. To support these different actors we foresee a system in which content can be represented at different degrees of formality. This will allow domain experts to create, review and modify models at a rather informal/human intelligible level and knowledge engineers to check the quality of the formal definitions and their correspondence with the informal parts they intend to represent. In order not to increase the overhead of human work, translation between different levels of formality should be as much automatised as possible. To support a coherent development and integration of the different components of the enterprise model we also foresee a tool in which all the relevant aspects of an enterprise can be modelled in a collaborative and cooperative manner, thus exploiting the synergy of "having to think the same thing out only once". To meet this vision we are developing MoKi (Modelling Wiki), a collaborative tool that (i) supports access to the enterprise model at different levels of formality (informal, semi-formal and formal), (ii) supports integrated modelling of several aspects of an enterprise, and (iii) ensures a coherent development of the formal part. In the current implementation of MoKi, we focus on an enterprise model describing the domain, the processes and the competencies of an enterprise. The choice of these aspects was originally motivated by the EU-project APOSDLE described in Section 3.

\section{Key Technology: Semantic Wiki}

MoKi is a tool based on Semantic MediaWiki (SMW) 2]. It extends SMW offering specific support for enterprise modelling. The choice of developing MoKi on top of a semantic wiki was made for several reasons. First, wikis are a state of the art collaborative tool. Second, users are quite familiar with wikis and editing of wiki pages, due to the growing popularity of wiki-based web sites. Third, it provides a uniform tool for the (informal) specification of the different components of an enterprise model (domain, processes, and competencies in our case). Fourth, the natural language descriptions inserted in a semantic wiki can be structured according to predefined templates, with the help of semantic constructs like attributes and relations. As a consequence, the informal descriptions in natural language contain enough structure to be automatically translated in formal models, thus allowing the reuse of informal descriptions for automatic ontology creation. Finally only a web-browser is required on the end user side to use the system.

Model elements are associated to MoKi pages which contain informal but structured information about the element itself. The typical page contains (i) an informal description of the element considered in natural language (images or drawings can be used as well), and (ii) a structured part, where the element is described by means of triples of the form (subject, relation, object), with the element itself playing the role of the subject. This natural language based, but also structured, description provides a natural bridge between formal and informal representation of knowledge. The user fills a page via forms, so he/she does not 
Modify concept: Conference

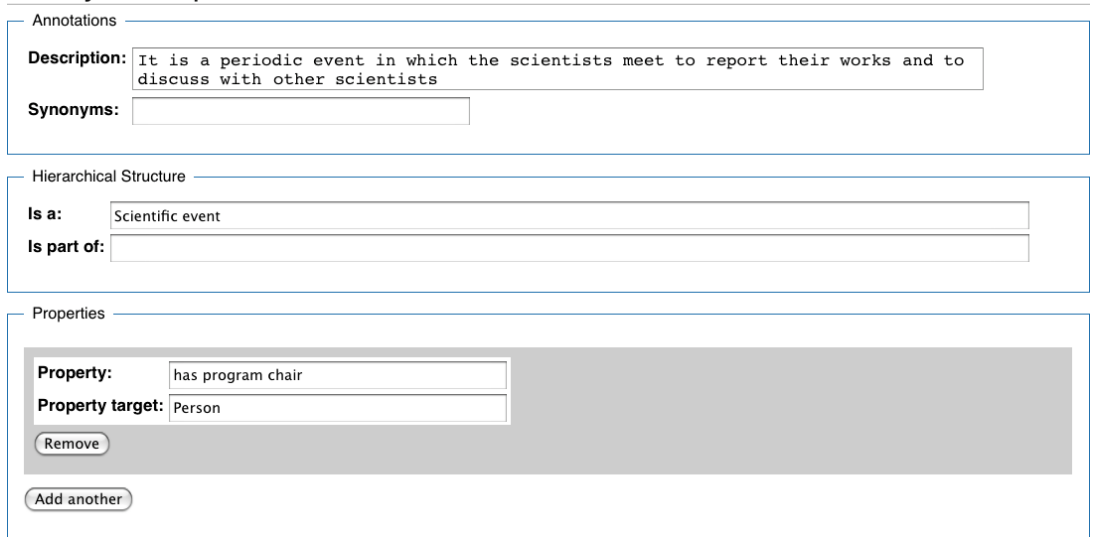

Fig. 1. An example of MoKi page

need to know any particular syntax or language to participate in the creation of the enterprise model. All the actors involved in the modelling activities can also interact with each others and exchange further ideas and comments using the discussion SMW's built-in functionality. An example of a MoKi page is shown in Figure 1. To support modelling, MoKi provides groups of functionalities, described below, that can be accessed via a wiki's style menu bar 1

Import. We provide two types of import functionalities. First, functionalities to import pre-existing structured knowledge. Currently it is possible to upload OWL domain ontologies, and to import list of domain concepts or tasks organized in a simple textual structure where indentation is used to indicate a hierarchy. Depending on the user's choice, the hierarchy is interpreted as taxonomy or partonomy. The import functionality takes care of creating the indicated concepts as well as the properties denoting the desired hierarchy. Second, text analysis functionalities such as the extraction of relevant terms from digital resources and the clustering of such terms according to their relatedness are included to support the utilization of available unstructured knowledge relevant for the modelling activity. The latter functionalities are provided by the KnowMiner [3], an advanced text analysis tool developed by the Know-Center.

Model Management. This set of functionalities supplies the basic functionality each modelling tool necessarily provides: creating, editing and deleting model elements. Depending on the type of element, pre-defined templates are loaded when it is created or edited. Such templates contain for instance properties for specifying a taxonomy or partonomy, or a sequence in the case of tasks.

Visualization. These functionalities allow to produce different types of graphical overviews of the models: they help the actors to deal with the global picture

${ }^{1}$ In what follows, we indicate with "model element" any basic component of the models (e.g. a concept, a relation, a task, a competency and so on). 
on the models and not only with the single model elements. For example, with the help of the IsA Browser and the PartOf Browser, the user can easily visualize and rearrange via drag 'n' drop the taxonomy and partonomy of concepts in the domain model.

Export. These functionalities support the fully automatic export of knowledge of the enterprise model into standard knowledge representation languages. At the moment the supported formalism is OWL for the whole enterprise model. On-going work is devoted to the addition of formal languages especially for task/process specification, where a number of standards already exist.

MoKi is built to facilitate the plugging-in of new or existing state-of-theart tools as we have done with KnowMiner. We plan to use this feature to add further functionalities in the future (e.g. tools providing knowledge elicitation techniques like card-sorting or laddering). Concerning the informa/semiformal/formal structuring of a page we currently focus on the semi-formal part, which also contains some informal description. A demo version of MoKi can be tried out on-line at moki.fbk.eu. A detailed description of the current version of MoKi is contained in the MoKi manual, available at the MoKi web site.

\section{Use Cases and Application}

MoKi has been successfully applied within the EU-project APOSDLE (See WWW . aposdle.org). Within this context, MoKi has been used to develop enterprise models in the following domains: environmental consultancy, electromagnetism simulation, innovation and knowledge management, requirements engineering, and statistical data analysis. Some of the experiences of an early usage of the system are described in [4]. In addition MoKi is used in applications that go beyond typical enterprise modelling: the representation of medical guidelines encoded in the ASBRU language (as part of the OncoCure project [5]), and the collection of data for the Personal Health Record of the Province of Trento, Italy (as part of the TreC project, see trec.fbk.eu). In each of these cases, MoKi has been applied in the context of a collaborative paradigm as described in [6].

\section{System Demonstration}

In the live demonstration we will present the main MoKi features that can be used to develop an enterprise model. We will first show how pages describing entities of the enterprise model look like, and we will describe the basic editing functionalities (add, edit, delete, rename). Then, we will demonstrate the import functionalities, starting from the domain model import. We will show that it is possible to create and pre-fill (annotations, class subclass/partof relations) pages in MoKi by importing an already available ontology. Then we will show that if there is an already available list of domain concepts (tasks) organized via plain-text indentation according to a taxonomy or partonomy (task/subtask decomposition), it it is possible to load pre-filled pages in MoKi preserving this kind of hierarchical structure. We will also present the term extraction functionality 
by uploading a digital resource (a pdf or MS Word document) to the MoKi and retrieving a list of candidate concepts, from which we will choose some to add as entities in our enterprise model. As regards the visualisation functionalities, we will demonstrate how to get different overviews of the models: we will show (i) how to display (and edit) in a tree-view the taxonomy or partonomy hierarchy of the element of the domain model, and (ii) how to display in a tabular-view the list of all elements (and their main properties) in the models. Finally, we will demonstrate that the enterprise model (its components) designed in MoKi can be exported into an OWL formal model, and later refined with any OWL ontology editor.

\section{Conclusions}

In this demonstration we have presented a new collaborative tool, called MoKi, to support the creation of integrated enterprise models. MoKi supports collaboration in the modelling activities among people with different skills and levels of expertise. We are currently improving the tool in several directions, which span from including support for the modelling of competencies, improving support for processes modelling and processes visualization, and improving the preservation of the alignment between the content described in MoKi pages and the formal models created.

Acknowledgements. We thank all the people involved in the modelling activities within the APOSDLE project for their useful suggestions and feedback. APOSDLE is partially funded under the FP6 of the EU Commission within the IST work program (FP6-IST-2004-027023). The Know-Center is funded by the Austrian Competence Center program Kplus under the auspices of the Austrian Ministry of Transport, Innovation and Technology and by the State of Styria.

\section{References}

1. Fox, M.S., Grüninger, M.: Enterprise modeling. AI Magazine 19(3), 109-121 (1998)

2. Krotzsch, M., Vrandecic, D., Volkel, M.: Wikipedia and the semantic web - the missing links. In: Proc. of the 1st Int. Wikimedia Conference, Wikimania 2005 (2005)

3. Pammer, V., Scheir, P., Lindstaedt, S.: Two protégé plug-ins for supporting document-based ontology engineering and ontological annotation at document-level. In: 10th International Protégé Conference, Budapest, Hungary, July 15-18 (2007)

4. Christl, C., Ghidini, C., Guss, J., Pammer, V., Rospocher, M., Lindstaedt, S., Scheir, P., Serafini, L.: Deploying semantic web technologies for work integrated learning in industry. a comparison: Sme vs. large sized company. In: Sheth, A., et al. (eds.) ISWC 2008. LNCS, vol. 5318, pp. 709-722. Springer, Heidelberg (2008)

5. Eccher, C., Ferro, A., Seyfang, A., Rospocher, M., Miksch, S.: Modeling clinical protocols using semantic MediaWiki: the case of the Oncocure project. In: ECAI workshop on Knowledge Management for Healthcare Processes, K4HelP (2008)

6. Rospocher, M., Ghidini, C., Serafini, L., Kump, B., Pammer, V., Lindstaedt, S.N., Faatz, A., Ley, T.: Collaborative enterprise integrated modelling. In: SWAP. CEUR Workshop Proceedings, vol. 426 (2008) 